\title{
Complicaciones de la Colecistectomía Videolaparoscópica en Cirugías electivas Hospital Militar Central de las FFAA Servicio de Cirugía General. Periodo Enero 2019 a Junio del 2020
}

\author{
Complications of Videolaparoscopic Cholecystectomy in elective surgeries \\ Central Military Hospital of the FFAA General Surgery Service. \\ Period January 2019 to June 2020
}

\author{
${ }^{\star}$ Hernando Raúl Cardozo Arias ${ }^{1},{ }^{\star}$ Cynthia Patricia Verdecchia Insfran ${ }^{1},{ }^{\star}$ Alejandro Caballero ${ }^{1}$, \\ ${ }^{\star}$ Amanda Fretes ${ }^{1},{ }^{\star *}$ Gloria Portillo ${ }^{1}{ }^{* *}$ Gustavo Roa $^{1}$
}

${ }^{1}$ Hospital Militar Central de las FFAA, Cirugía General. Paraguay

\section{RESUMEN}

Sin duda alguna, el procedimiento laparoscópico más utilizado en el mundo lo constituye la colecistectomía, pero a pesar de ser unas de las técnicas sofisticadas el riesgo de complicaciones sigue siendo elevada. El objetivo del estudio fue Determinar las complicaciones quirúrgicas de los pacientes sometidos a colecistectomía laparoscópica electiva del Hospital Militar Central durante el periodo enero del 2019 a junio del 2020, El estudio fue observacional, descriptivo, prospectivo, con muestreo no probabilístico de casos consecutivos, de pacientes admitidos al servicio de cirugía general del Hospital Militar Central sometidos a colecistectomía videolaparoscopica durante el periodo arriba mencionado. La muestra correspondió a 371 fichas de pacientes operados. La complicación quirúrgica fue de $1,06 \%$. Las edades de los pacientes se encontraban entre 15 a 39 años en un $36,81 \%$ y $51,28 \%$ de los pacientes fueron del sexo femenino. Las complicaciones durante el desarrollo de la colecistectomía fueron dos casos de hemorragias en el lecho operatorio que se convirtió al final en una cirugía abierta y las complicaciones que se originaron en el período postoperatorio inmediato se encontraron el bilioperitoneo y fuga del clip de la arteria cística. copia.

Palabras clave: Complicaciones, pacientes, colecistectomía laparos-

\section{ABSTRACT}

Undoubtedly, the most widely used laparoscopic procedure in the world is cholecystectomy, but despite being one of the sophisticated techniques, the risk of complications remains high. The objective of the study was to determine the surgical complications of patients undergoing elective laparoscopic cholecystectomy at the Central Military Hospital during the period January 2019 to June 2020, The study was observational, descriptive, prospective, with non-probability sampling of consecutive cases, of patients admitted to the general surgery service of the Central Military Hospital undergoing videolaparoscopic cholecystectomy during the period mentioned above. The sample corresponded to 371 operative patient files. The surgical complication of patients undergoing elective laparoscopic cholecystectomy was $1.06 \%$. The ages of the patients were between 15 to 39 years old in $36.81 \%$ and $51.28 \%$ were female. Complications during the development of cholecystectomy were two cases of hemorrhages in the operative bed that eventually turned into open surgery, and complications that originated in the immediate postoperative period included bilioperitoneum and leakage of the clip of the cystic artery.

Key words: Complications. Patients. Laparoscopic cholecystectomy.

\section{INTRODUCCIÓN}

La colecistectomía laparoscópica se realiza de manera rutinaria desde finales de los años ochenta y en la actualidad, se considera como el método de referencia para el tratamiento de las patologías biliares ${ }^{(1)}$.

Entre las complicaciones podemos citar la lesión de los vasos de la pared abdominal, lesión de vísceras hueca y maciza, clipaje y sección total o parcial del colédoco, hemorragia en el triángulo de Calot, lesión con el electrocauterio. Estas complicaciones pueden presentarse como biliomas, fístulas, ictericia, peritonitis, etc., dependiendo de la lesión causada, y que haya pasado desapercibida en el acto operatorio ${ }^{(2)}$.

La colecistectomía laparoscópica ha sustituido a la cirugía

* Residente de Cirugía General - Hospital Militar Central de las FFAA

** Médico de Planta Servicio de Cirugía General

Autor correspondiente: Dr. Raúl Cardozo Arias - Email: raulcardozoarias@gmail.com

Recibido: 27/07/2020 - Aceptado: 21/10/2020

(c) Es Este es un artículo publicado en acceso abierto bajo una licencia Creative Commons 
abierta en el tratamiento de elección de los pacientes con colelitiasis, dado que, con esta, los pacientes presentan una menor estancia hospitalaria, menor dolor postoperatorio y menor número de complicaciones postoperatorias ${ }^{(3)}$.

$\mathrm{Al}$ igual que en todos los procedimientos quirúrgicos, es importante el desarrollo de una técnica adecuada, estandarizada, reproducible, basada en unos lineamientos y estrategias ya establecidas, que permita la sistematización del procedimiento. No quiere decir lo anterior que debe existir una técnica mundial, sino que cada cirujano debe, por sí mismo, adoptar una técnica que le permita sentirse cómodo y minimizar el riesgo de complicaciones $^{(4)}$.

En un estudio realizado por Priego y colaboradores arrojaron los siguientes resultados donde la estancia media postoperatoria fue de 3,06 días. La tasa de conversión a cirugía abierta de $8,3 \%$ y la de complicaciones quirúrgicas mayores del 2,34\%, siendo la más frecuente el hemoperitoneo (1\%). Se produjeron 13 lesiones de la vía biliar durante la cirugía laparoscópica $(0,3 \%), 51$ pacientes $(1,3 \%)$ fueron re intervenidos precozmente tras la cirugía (hemo-/coleperitoneo) y fallecieron un total de 5 pacientes $(0,13 \%)$. Los resultados deben interpretarse con reservas al tratarse de un estudio retrospectivo y sujeto a variables no controladas (elevado número de cirujanos y curva aprendizaje continuada). Llama la atención la menor tasa de conversión y estancia post-operatoria en la primera década de la curva de aprendizaje, aunque podría estar en relación con la mayor selección de los pacientes y con el menor número de colecistitis realizadas por vía laparoscópica en el comienzo de la serie ${ }^{(5)}$.

Complicaciones durante el desarrollo de la colecistectomía.

Lesión de vía biliar: Clipaje y sección total o parcial del colédoco, esta complicación tiene lugar al traccionar excesivamente del cístico angulando el colédoco y colocando el clip de forma que se secciona la vía biliar principal en lugar del cístico ${ }^{(6)}$.

Hemorragia en el triángulo de Calot.

Lesión con el electrocauterio que pueden producir zonas de necrosis o retracciones que condicionen posteriormente una estenosis de la vía biliar ${ }^{(7)}$.

Las lesiones de la vía biliar principal han supuesto desde la aparición de la era laparoscópica la mayor de las preocupaciones de los diferentes autores, unos para desprestigiar la técnica y otros para intentar que las cifras de dichas lesiones no sobrepasaran las consideradas como patrón en las grandes series de la cirugía laparotómica y que oscilan entre el 0,1 y el $0,5 \%{ }^{(8)}$.

Las infecciones de las heridas son raras y de poca relevancia clínica, pudiéndose solucionar de forma sencilla con el tratamiento adecuado.

Hemorragia:

- Las dos causas más frecuentes de hemorragia en el transcurso de la colecistectomía laparoscópica son las lesiones de la arteria cística y el sangrado del lecho vesicular

- Lesión de los vasos de la pared abdominal, esta complicación es relativamente frecuente, se produce fundamentalmente con la introducción de los trocares y en especial con aquellos que poseen aristas cortantes, Lesión de víscera hueca, esta puede ser en estómago, intestino delgado o colon. Lesión de víscera maciza es infrecuente, se descubre una vez introducida la óptica y no sue- le revestir gravedad ya que suele tratarse de punciones superficiales del hígado, que paran espontáneamente de sangrar $^{(9)}$.

\section{MATERIALES Y MÉTODOS}

Estudio observacional, descriptivo, Prospectivo, con muestreo no probabilístico de casos consecutivos de pacientes sometidos a colecistectomía laparoscópica electiva en el Servicio de Cirugía del Hospital Militar Central durante el periodo enero del 2019 a Junio del 2020, Para el análisis de los datos, se utilizó el Paquete estadístico de Epi Info 7.2, se analizaron medidas de tendencia central, de dispersión y posterior a eso los datos se presentaron en gráficos para su discusión.

\section{RESULTADOS}

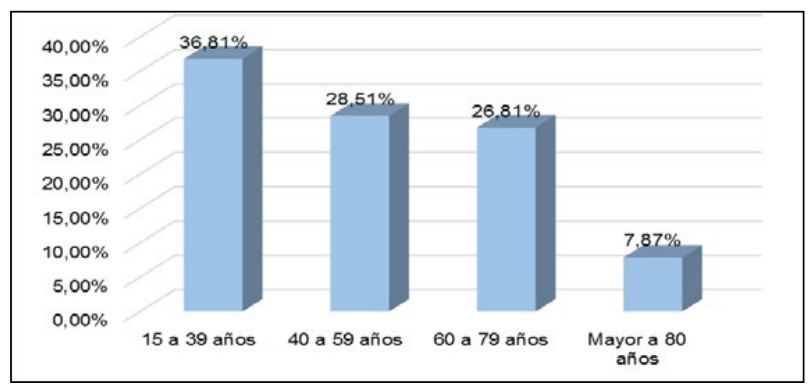

Fuente: archivo de fichas clínicas Hospital Militar Central

Gráfico 1. Distribución porcentual del grupo etáreo de los pacientes. $(n=371)$

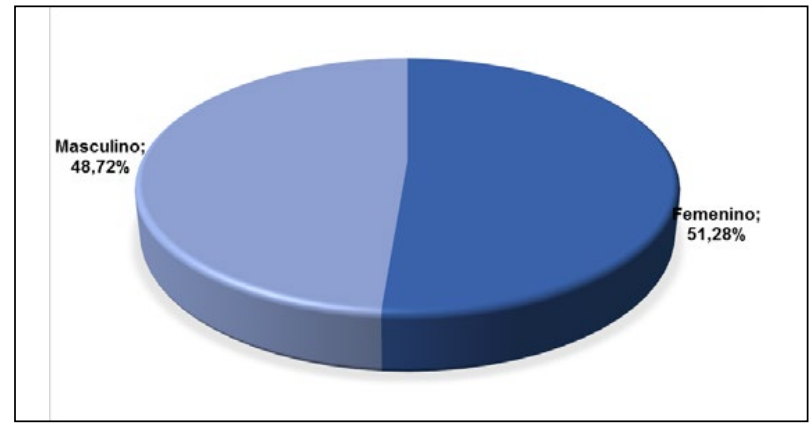

Fuente: archivo de fichas clínicas Hospital Militar Central

Gráfico 2. Distribución porcentual de los pacientes según sexo. $(n=371)$

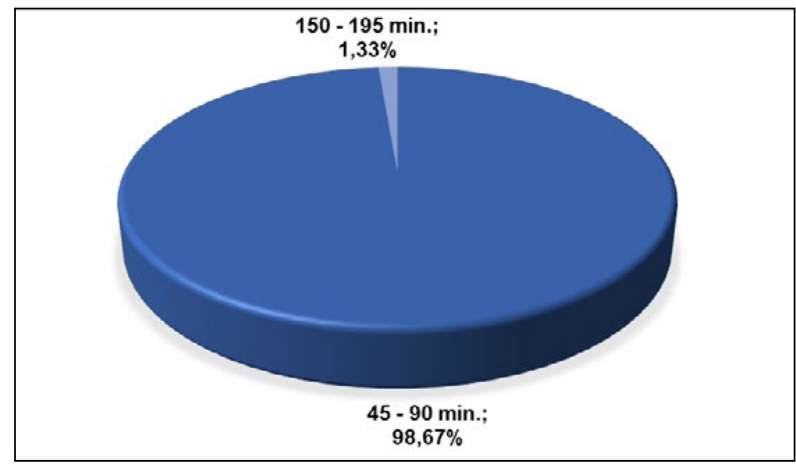

Fuente: archivo de fichas clínicas Hospital Militar Central

Gráfico 3. Distribución porcentual del promedio de tiempo quirúrgico de los pacientes sometidos colecistectomía laparoscópica. $(n=371)$ 


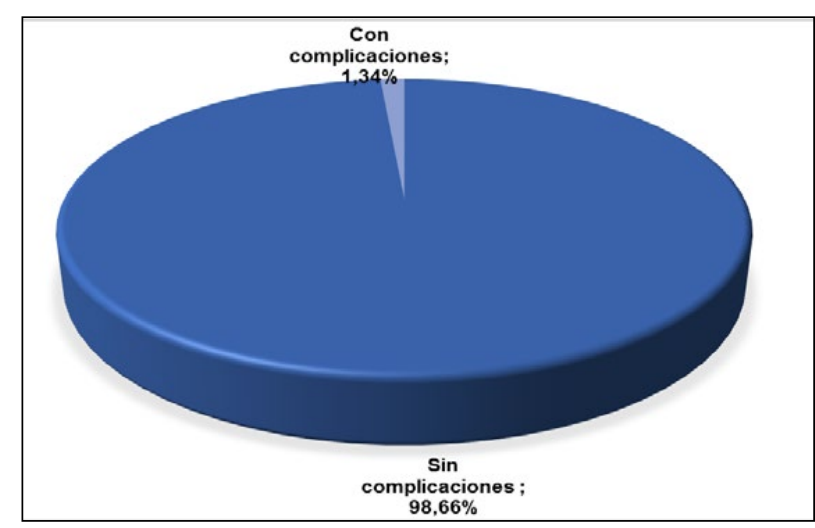

Fuente: archivo de fichas clínicas Hospital Militar Central

Gráfico 4. Distribución porcentual de complicaciones de los pacientes sometidos colecistectomía laparoscópica. $(n=371)$

Tabla 1. Distribución numérica y porcentual según aparición de complicaciones

\begin{tabular}{|l|c|c|}
\hline \multicolumn{1}{|c|}{ Complicaciones } & Frecuencia & $\%$ \\
\hline $\begin{array}{l}\text { Complicaciones propias } \\
\text { relacionadas con el método. }\end{array}$ & & \\
\hline $\begin{array}{l}\text { Lesión del parénquima hepático } \\
\text { Hemoperitoneo por sección de los } \\
\text { vasos de epiplón mayor }\end{array}$ & 1 & 50 \\
\hline $\begin{array}{l}\text { Complicaciones durante el } \\
\text { desarrollo de la colecistectomía. }\end{array}$ & & 50 \\
\hline $\begin{array}{l}\text { Hemoperitoneo por fuga del clip de la } \\
\text { arteria cística }\end{array}$ & 1 & 50 \\
Sangrado del lecho hepático & 1 & 50 \\
\hline $\begin{array}{l}\text { Complicaciones que se originaron } \\
\text { en el período postoperatorio } \\
\text { inmediato. }\end{array}$ & & \\
\hline Bilioperitoneo & 2 & 67 \\
\hline
\end{tabular}

Fuente: archivo de fichas clínicas Hospital Militar Central

\section{DISCUSIÓN Y COMENTARIOS}

Las complicaciones quirúrgicas de los pacientes sometidos a colecistectomía laparoscópica electiva del Hospital Militar Central durante el periodo de enero del 2019 a junio del 2020, fue de $1,06 \%$, datos que coincide con el realizado por Priego y colaboradores que es de $2,34 \%$ de complicaciones ${ }^{(2)}$. Díaz y colaboradores en cambio encontraron complicaciones en 3,7 \% de los pacientes ${ }^{(10)}$.
Las edades de los pacientes se encontraban entre 15 a 39 años en un $36,81 \%$ y no se encontraron mucha diferencia en el sexo donde el $51,28 \%$ de los pacientes son del sexo femenino y el $48,72 \%$ masculino. Se corroboraron estudios similares donde la edad promedio fue de 46 años ( 7 a 88 años) y $67 \%$ de ellos del sexo femenino ${ }^{(10)}$. El tiempo quirúrgico fue de 45 a $90 \mathrm{~min}$. en un $98,67 \%$ en el estudio realizado. En el estudio realizado por Fernández el tiempo quirúrgico promedio fue de 51,2 min. La estancia hospitalaria media fue $25,7 \mathrm{~h}$. En el $76,92 \%$ de los pacientes el ingreso fue menor de $24 \mathrm{~h}$. No hubo ningún reingreso ni complicación intraoperatoria o postoperatoria importante $e^{(6)}$. El presente estudio se encontró una Lesión del parénquima hepático y hemoperitoneo por sección de los vasos de epiplón mayor que son lesiones propias del método, entre las lesiones específicas de la colecistectomía se encontró un paciente que tuvo hemoperitoneo por fuga del clip de la arteria cística y sangrado de lecho hepático y de las complicaciones que se originaron en el período postoperatorio inmediato se encontró dos casos de Bilioperitoneo. En otros estudios se describen complicaciones posquirúrgicas, como hemoperitoneo, bilioperitoneo y colecciones intraabdominales, con incidencia de $0,14 \%, 0,1 \%$ y $0,04 \%$, respectivamente. En el $60 \%$ de las complicaciones aparecieron en el postoperatorio, datos que coinciden con el de Ibáñez ${ }^{(11)}$.

\section{CONCLUSIONES}

- Las complicaciones quirúrgicas de los pacientes sometidos a colecistectomía laparoscópica electiva del Hospital Militar Central durante el periodo enero del 2019 a junio del 2020 fue de $1,06 \%$.

- El tiempo quirúrgico fue de 45 a 90 min. en un $98,67 \%$ en el estudio realizado.

- Las edades de los pacientes se encontraban entre 15 a 39 años en un $36,81 \%$ y no se encontraron mucha diferencia en el sexo donde el $51,28 \%$ de los pacientes son del sexo femenino y el $48,72 \%$ masculino.

- Con respecto a las complicaciones propias relacionadas con el método, se encontró lesiones de vísceras macizas y lesión de vasos.

- Según las complicaciones durante el desarrollo de la colecistectomía se encontró dos casos de hemorragias en el lecho operatorio y en el triángulo de calot que se convirtió al final en una cirugía abierta.

- Complicaciones que se originaron en el período postoperatorio inmediato se encontró el bilioperitoneo. 


\section{REFERENCIAS BIBLIOGRÁFICAS}

1. Tenconi SM, Boni L, Colombo EM, Dionigi G, Rovera F, Cassinotti E. Laparoscopic cholecystectomy as day-surgery procedure: Current indications and patients. Int J Surg. 2008; 6 (Suppl.1): S86-8.

2. Delgado Gomisa F, Blanes Massonb S, Gómez Abrilc J, Richart Aznard R. Trullenque Juand R. Complicaciones de la cirugía laparoscópica; 2001; 69 (3): 197-336. Recuperado de: http://www.elsevier.es/es-revista-cirugiaespanola-36-articulo-complicaciones-cirugia-laparoscopica-12003373

3. Barrios C. Cirugías con laparoscopia en Paraguay. Periódico ABC digital. 2017. Recuperado de: http://www.abc.com.py/edicion-impresa/suplementos/salud/cirugias-con-laparoscopia-1619361.html

4. Von Bernhardi R, Zanlungo S, Arrese M, Arteaga A. El síndrome metabólico: De factor agravante a principal factor de riesgo patogénico en diversas enfermedades crónicas. Rev. méd. Chile [Internet]. 2010 Ago [citado 2018 set 13]; 138(8): [Aprox. 7p.]. Disponible en: http://www.scielo.cl/ scielo.php?script=sci_arttext\&pid=S0034-98872010000800012\&lng=es

5. Priego P, Ramiro C, Molina JM, Rodríguez Velasco G, Lobo E, Galindo J, et al. Resultados de la colecistectomía laparoscópica en un hospital universitario de tercer nivel tras 17 años de experiencia. Rev Esp Enfermedades Dig. 2009 Jan;101(1):20- 30.

6. Galloso CGL, Frías JRA, Pérez BO, Petersson RM, Benavides GS. Factores que influyen en la conversión de la colecistectomía video laparoscópica a cirugía tradicional. Rev Cub Med Mil 2012; 41 (4). Disponible en: http:// www.medigraphic.com/cgi-bin/new/resumen.cgi?IDARTICULO=40657
7. Galloso Cueto GL, Frías Jiménez R. Consideraciones sobre la evolución histórica de la cirugía laparoscópica: colecistectomía. Rev. Med. Electrón. [Internet]. 2010 Dic [citado 2018 ago 14] ; 32(Suppl 7). Disponible en: http://scielo.sld.cu/scielo.php?script=sci_arttext\&pid=S1684$18242010000700004 \& \operatorname{lng}=\mathrm{es}$.

8. Álvarez LF, Rivera D, Esmeral ME, García MC, Toro DF, Rojas OL. Colecistectomía laparoscópica difícil, estrategias de manejo. Revista Colombiana de Cirugía [Internet]. 2013; 28 (3):186-95. Disponible en: http:// www.redalyc.org/articulo.oa?id $=355535158002$

9. Delgado Gomisa F, Blanes Massonb S, Gómez Abrilc J, Richart Aznard R. Trullenque Juand R. Complicaciones de la cirugía laparoscópica; 2001. 69 (3) 197-336. Recuperado de: http://www.elsevier.es/es-revista-cirugiaespanola-36-articulo-complicaciones-cirugia-laparoscopica-12003373

10. Tenconi SM, Boni L, Colombo EM, Dionigi G, Rovera F, Cassinotti E. Laparoscopic cholecystectomy as day-surgery procedure: current indications and patients' selection. Int J Surg. 2008; 6 Suppl 1:S86-8. doi: 10.1016/j.ijsu.2008.12.032. Epub 2008 Dec 14.

11. González Sosa G, Díaz Mesa J, Collera Rodríguez S, González García GA, More Vara S, Romero Rodríguez J. Îleo biliar: complicación poco frecuente de la litiasis vesicular. Rev Cubana Cir [Internet]. 2010 Jun [citado 2018 Jul 07]; 49(2). Disponible en: http://scielo.sld.cu/scielo. php?script=sci_arttext\&pid=S0034-74932010000200011\&lng=es\&nrm= iso\&tlng $=\mathrm{es}$ 